\title{
Théologiques
}

Théologiques

\section{La mort et le rien}

\section{Fernand Couturier}

Volume 4, numéro 2, octobre 1996

\section{L’épreuve du rien}

URI : https://id.erudit.org/iderudit/602438ar

DOI : https://doi.org/10.7202/602438ar

Aller au sommaire du numéro

Éditeur(s)

Faculté de théologie de l'Université de Montréal

ISSN

1188-7109 (imprimé)

1492-1413 (numérique)

Découvrir la revue

\section{Citer cet article}

Couturier, F. (1996). La mort et le rien. Théologiques, 4(2), 11-39.

https://doi.org/10.7202/602438ar

\section{Résumé de l'article}

Ce texte se veut une explicitation simple d'une parole énigmatique de Heidegger : La mort est l'écrin du rien. Il invite à une lecture du texte original qui a besoin de prendre la parole avant le débit des discussions de second degré. Des « scientifiques " s'en trouveront frustrés. Mais tout esprit attentif pourra entrevoir à partir de l'oeuvre même comment la mort, assumée véritablement dans la vie, tout en affichant impitoyablement le rien des choses laisse se profiler l'être, et partant introduit la pensée dans le dépaysement de l'étrange, de l'originel, de ce qui ne renvoie plus à autre chose comme à son fondement. Cet abîme du rien entretient l'interrogation et dispense à la pensée l'ouverture d'une inquiétude salvatrice. d'utilisation que vous pouvez consulter en ligne.

https://apropos.erudit.org/fr/usagers/politique-dutilisation/ 


\title{
La mort et le rien
}

\section{Fernand COUTURIER}

\author{
Der Tod ist der Schrein des Nichts \\ La mort est l'écrin du rien.
}

\section{RÉSUMÉ}

Ce texte se veut une explicitation simple d'une parole énigmatique de Heidegger : La mort est l'écrin du rien. Il invite à une lecture du texte original qui a besoin de prendre la parole avant le débit des discussions de second degré. Des "scientifiques " s'en trouveront frustrés. Mais tout esprit attentif pourra entrevoir à partir de l'œuvre même comment la mort, assumée véritablement dans la vie, tout en affichant impitoyablement le rien des choses laisse se profiler l'être, et partant introduit la pensée dans le dépaysement de l'étrange, de l'originel, de ce qui ne renvoie plus à autre chose comme à son fondement. Cet abîme du rien entretient l'interrogation et dispense à la pensée l'ouverture d'une inquiétude salvatrice.

This text works towards a simple explanation of an enigmatic speech by Heidegger : La mort est l'écrin du rien. It invites a reading of the original text that needs to speak before the flow of second order discussions. "Scientists * will find themselves frustrated. But all attentive minds can glimpse from the work itself, how death, genuinely accepted in life, though mercilessly announcing nothingness, left to emanate being and hence introduces thinking in the disorientation of the strange, of the original, of that which no longer refers to something else, like its foundation. This abyss of nothingness maintains a questioning and offers to a thinking the opening of a saving anxiety.

Poésie par l'image évocatrice, pensée par la rigueur cachée. Les deux comme réponse à une même inspiration : l'interpellation de l'être. Nous nous proposons une interprétation de cette parole énigmatique de Heidegger prononcée dans la conférence intitulée : La chose. Comme un défi de parler sans trop de complexité de l'immédiat et du simple tout en lui promettant fidélité. 
Il faut d'abord replacer la parole citée dans son contexte immédiat pour prendre une juste mesure de son caractère énigmatique, et y trouver, peut-être, quelque indice pour commencer l'interprétation.

Der Tod ist der Schrein des Nichts, dessen nämlich, was in aller Hinsicht niemals etwas blo $\beta$ Seiendes ist, was aber gleichwohl west, sogar als das Geheimnis des Seins selbst ${ }^{1}$.

La mort est l'écrin du rien, à savoir de ce qui à tout point de vue n'est jamais quelque chose de simplement étant, mais qui cependant se déploie, voire comme le secret de l'être lui-même.

Le choix du mot écrin pour Schrein est déjà une interprétation. Schrein peut se traduire de multiples manières : châsse, cercueil, armoire, coffre, arche, coffret, écrin. C'est la référence du rien au secret de l'être qui nous fait choisir le vocable écrin. Pourquoi? En raison de la nature et de la fonction de cette chose qu'est un écrin.

L'écrin est un coffret empreint de singularité. Il est souvent fait d'un matériau plutôt recherché. L'écrin, en lui-même, se singularise et retient l'attention. Une attention qu'il ne garde pas pour lui, cependant, mais qu'il oriente vers son contenu. D'emblée l'écrin attire la pensée vers ce qu'il renferme et contient: une chose spéciale et d'une certaine noblesse. Dans un écrin, on serre un joyau. L'écrin l'abrite et en assure la garde.

Et qu'en est-il du joyau? Il entretient l'intérêt et stimule la hâte d'ouvrir l'écrin. Tout d'or, d'argent ou de pierreries, le joyau étincelle et fascine. Mais il ne déploie vraiment son être, il ne se manifeste authentiquement que lorsque, sorti de l'écrin, il est porté par quelqu'un. Qu'il soit couronne, épingle, bague ou bracelet, il signifie une majesté, il rehausse une tenue, ou appuie une splendeur. Son rôle est d'illuminer une présence, de mettre en relief ce qui mérite d'être vu. Sa fascination au service de la grandeur et de la beauté. Le charme est son essence.

La mort est l'écrin du rien. Le rien aurait-il le caractère du joyau pour pouvoir être serré en écrin? Le rien, ne doit-on pas plutôt penser, n'a rien de la rutilance des pierres précieuses ni ne s'apparente de près ou de loin aux nobles métaux. Selon toutes les apparences, en effet, il n'a rien de cela. Mais l'essence du joyau, vient-on de souligner, est de charmer, c'està-dire de fasciner, de plaire, et en même temps de renvoyer à quelqu'un dont il annonce et éclaire la présence, la grandeur ou la beauté. Mais comment le rien peut-il fasciner, charmer et illuminer? Cela ne dépasse-t-

1 Vorträge und Aufsätze. Tübingen, Neske Pfullingen, 1954, p. 177. Voir Essais et conférence, Paris, Gallimard, 1958, p. 212. 
il pas l'entendement le plus normal? Et vers quoi le rien pourrait-il orienter, si tant est que la capacité de renvoyer à autre chose lui appartienne de quelque façon? Le rien, semble-t-il, n'a vraiment rien de fascinant, n'a rien de charmant, n'a rien d'éclairant et ne favorise en rien la présence et le déploiement de quoi que ce soit.

Voilà le rien selon l'opinion courante qui, d'ailleurs, s'alimente ellemême aux apparences. Du rien, dit-elle, il n'y a rien à dire. Habituellement, quand on parle, on dit quelque chose à propos d'une chose ou l'autre, identifiable et désignable dans un environnement déterminé. Le rien, c'est entendu, n'est pas une telle chose. Parler du rien, c'est parler pour ne rien dire, c'est parler dans le vide et inutilement. Et sur ce, on croit la cause définitivement entendue.

Mais peut-on se fier à l'opinion courante pour la compréhension du rien? Car si, en effet, parler pour ne rien dire et dire le rien étaient des propos du tout au tout différents? Ainsi les humains, en s'abstenant de dire le rien, s'imposeraient peut-être une privation dont ils ne sauraient jamais apprécier la grandeur. Alors il faut demander : le rien constitue-t-il vraiment un hors-propos absolu? Tout ce dont on peut parler significativement doit-il être de l'ordre de la chose matérielle montrable et repérable dans l'espace et le temps? ou encore de l'ordre des réalités dites immatérielles et spirituelles, telles que la pensée, l'esprit, l'âme ou Dieu? Qu'en est-il, par exemple, de l'être? Jamais on ne rencontre l'être à côté ou parmi les choses qui sont données d'une manière ou d'une autre, qu'elles soient nommées pierre, fumée, vin, homme, chenille, pain, image, ou bien pensée, esprit, âme, ange et Dieu. Pourtant, on a tant et tant dit à son propos. En parlant de lui, a-t-on vraiment parlé pour ne rien dire de pertinent ou de sérieux? Et alors le rien, lui? Pourquoi ne pourrait-on pas parler aussi à propos du rien sans nécessairement être condamné à ne dire rien qui vaille? Pourquoi le rien ne pourrait-il pas constituer un propos sensé pour le langage?

D'abord, on pourrait demander à l'opinion courante sur quelles apparences elle se base pour déclarer qu'il n'y a rien à dire du rien. En effet, comment le rien peut-il avoir des apparences puisqu'il n'est rien? Car les apparences, comme il semble aller de soi, sont toujours apparences de quelque chose. Et voilà que pointe ainsi la nécessité de regarder autrement et ailleurs que vers les apparences si jamais on veut dire et comprendre quelque chose du rien. Car si, en effet, la manifestation était un phénomène qui déborde la simple présence des choses sur laquelle semblent se fonder les apparences? Si la manifestation comprenait aussi une sorte d'apparaître qui dépasse celui des simples apparences? 
Alors comment laisser apparaître le rien? Il suffit peut-être de simplement raviver l'attention. Le rien, peut-on tout de suite observer, s'est toujours déjà subrepticement introduit dans l'horizon des préoccupations et des propos de tous les jours. En effet, ne dit-on pas couramment : ça ne sert à rien, ça ne vaut rien, il est bon a rien, il ne fait rien, ça ne donne rien, il n'y a rien à faire, il n'y a rien à dire ou à redire, offrir un petit rien à quelqu'un, etc. Sans aucune apparence expresse ou explicite qui capte immédiatement l'attention ou le regard, il y a déjà une manifestation du rien dans ces manières toutes simples de dire. Puis l'opinion courante elle-même ne laisse-t-elle pas apparaître le rien en prétendant résolument qu'il n'y a rien à dire du rien? Et en plus, de leur côté, les gens de la science ${ }^{2}$, de la technologie et des affaires, en tant que tels, ne disent-ils pas qu'ils s'occupent de choses importantes, de choses sérieuses, de choses qui comptent, de choses qui sont véritablement, et qu'ils ne s'intéressent à rien d'autre? Tous se rapportent à des étants ${ }^{3}$ de quelque catégorie. Et en dehors de cela, à rien. Ainsi l'exige le sérieux de leurs entreprises. Mais dans ce cas encore, voilà que le rien se manifeste déjà avant même qu'on y réfléchisse expressément. Se pourrait-il, doit-on maintenant se demander, que le rien habite ainsi constamment le langage sans qu'il puisse éventuellement constituer un propos sensé, sans qu'on puisse parler sensément de lui? Ce serait pour le moins étonnant.

2 Pour cette partie sur le rien nous nous référons à Vittorio KLOSTERMANN,Was ist Metaphysik? Frankfurt, $7^{\mathrm{e}}$ édition. Noter que ce titre regroupe dans la plupart des éditions la Leçon inaugurale du même titre à l'Université de Freiburg en 1929, la Postface de 1943 et l'Introduction de 1949. Pour le moment nous nous en tenons à la Leçon inaugurale.

3 Étant. Participe présent, substantivé, du verbe être. Tout ce qui est est un étant. Substantivation nécessaire pour suivre la pensée de l'être heideggérienne. Même si cette tournure n'est pas courante en français et paraît étrange à l'oreille non encore habituée, il reste qu'elle est tout aussi normale et légitime que, par exemple, le nom habitant qui vient du participe présent de habiter, que représentant de représenter, participant de participer, vivant de vivre, mourant de mourir, combattant de combattre, calmant de calmer, stimulant de stimuler, irritant de irriter, trafiquant de trafiquer, tenant de tenir, aboutissant de aboutir, etc. Nous insistons à dessein parce que les lecteurs français de Heidegger résistent toujours à l'emploi du nom étant pour une chose qui est. Pourquoi? Peut-être parce que la philosophie traditionnelle et aussi, dans son sillage, le langage courant ont plutôt l'habitude de substantiver l'infinitif être. On parle de Dieu comme de l'être suprême. On dit aussi des humains qu'ils sont des êtres. Mais cette manière a pour inconvénient de ramener l'être à l'étant et, obnubilant ainsi leur différence, empêche la pensée de penser l'être en lui-même. Or ceci est le projet heideggérien. 
Il faut remarquer, cependant, que le rien du langage de tous les jours est traité comme une sorte d'objet. Toures les expressions relevées, en effet, peuvent être considérées comme apparentées à ces autres qui, elles, portent sur des choses déterminées : ça sert à quelque chose, ça vaut son pesant d'or, ça vaut la peine, il est bon à tout faire, il y a beaucoup à redire, etc. On le voit : les manières de dire qui parlent du rien modèlent leur tournure sur le langage qui parle des choses. Elles parlent du rien comme s'il était lui-même une chose ou un objet. Les choses, les étants sont. Mais le rien est-il vraiment? Comment être sans être une chose? Ces manières de dire évoquent le rien, mais l'effacent aussitôt, pour ainsi dire, en le présentant abusivement comme une chose qui est, comme ce qui lui est rigoureusement contraire, en somme, comme un étant qu'il ne saurait être. Et il importe de souligner aussi que le rien, que le monde de la science et celui des entreprises excluent de leur domaine d'intérêt, est également tout juste la contrepartie des choses tangibles, concrètes, comptables dont il font leur pain et miel. Ce rien est le contraire de ce qui est, de l'étant. Et dans la manière de dire, on le traite encore comme un objet dont on dit par ailleurs ne pas s'occuper.

Alors la logique se fait secourable et intervient pour lever l'ambiguité de la situation. Elle déclare le rien comme la simple négation de ce qui est, de tout ce qui est, de l'étant dans son ensemble. Le rien apparaît comme aboutissant de cette opération de l'entendement. Le rien n'est pas l'étant; il vient tout simplement de l'étant dans son ensemble en tant que nié. Le rien se range alors dans la catégorie du négatif et du nié. C'est sa détermination ultime. Selon la logique, le ne...pas, la négativité, la négation seraient la détermination dernière du rien. Mais à ce propos, Heidegger questionne ainsi : y a-t-il le rien seulement parce qu'il y a le ne...pas et la négation? Ou bien, au contraire, y a-t-il le ne...pas et la négation seulement parce qu'il y a le rien? Et il s'emploie à montrer que le rien est plus originel que la négation et son ne... pas.

Cette thèse suppose cependant que le rien se montre de quelque manière avant même que l'entendement ne procède à la négation; et qu'on doive pouvoir le rencontrer et l'apercevoir. Or c'est le cas, et on le peut. En dépit des limites relevées dans les manières quotidiennes de parler du rien comme de quelque chose ou d'un objet, même si le rien n'y est qu'à peine effleuré, il reste qu'il y est donné d'une certaine façon. Ce rien, pourrait-on résumer, est le tout autre de la totalité de l'étant. Mais pour qu'il apparaisse ainsi, il faut d'abord que cette totalité nous soit donnée. Or l'entendement humain est fini et il ne saurait saisir en ellemême la totalité de l'étant du présent, du passé et de l'avenir. Le mieux qu'il puisse faire, semble-t-il, c'est d'avoir l'idée de cette totalité et de la nier. Mais cela ne donne qu'un concept formel du rien et non pas le rien 
en lui-même. Et de toute manière ce rien résulterait encore de la négation. Y aurait-il une autre manière d'accéder à la totalité de l'étant que celle de sa saisie par l'entendement? Il y en a une, en effet.

Il y a une différence essentielle entre saisir la totalité de l'étant en elle-même (Erfassen des Ganzen des Seienden an sich, p. 30) et se trouver au milieu de l'étant dans son ensemble (Sich befinden inmitten des Seienden im Ganzen, p. 30). Il se trouve chez l'humain différentes humeurs qui révèlent le tout des choses dans une certaine unité. Comme l'ennui profond, par exemple. "Tout m'ennuie", laisse-t-on parfois échapper. La joie peut aussi dévoiler le tout. Par exemple, la présence d'une personne aimée transfigure et illumine toute la réalité. Ces tonalités affectives qui manifestent la totalité des choses en nous situant en elles et en nous y accordant (Befindlichkeit der Stimmung, p. 31) selon une humeur déterminée viennent d'un trait fondamental de notre $\mathrm{Da}$-sein ${ }^{4}$, de notre caractéristique propre d'humain d'être le là de l'être. Cet être en accord fondamental avec les choses ou les étants s'appelle le sentiment de situation (Befindlichkeit). Mais, faut-il observer, ces manières de dévoiler l'étant dans l'ensemble cachent précisément le rien. Alors que faire et que dire?

Encore une fois il faut renoncer au subterfuge logique pour faire apparaître le rien à partir de la négation de l'étant dans l'ensemble manifesté par ces humeurs. Cela ferait changer de registre et ramènerait au plan de la détermination du rien par la négativité résultant de cette opération de l'entendement qu'est la négation. Alors, pour préserver la rigueur de la démarche, il faut demander s'il n'y aurait pas une humeur qui, tout en nous situant au milieu de l'étant dans son ensemble, pourrait faire que nous nous trouvions devant le rien lui-même. Il y a effectivement une telle humeur, et c'est l'angoisse.

L'angoisse, une humeur fondamentale. Il faut la distinguer de l'anxiété fébrile et de surface qui suinte du caractère craintif, pusillanime et peureux. L'angoisse est autre chose que la peur. Celle-ci s'agite et prend panique devant une chose déterminée qui représente un danger. Par exemple, j'ai peur de la chaussée glacée, j'ai peur pour ma voiture et surtout pour moi-même. Alors je m'efforce de bien tenir la route et je m'agrippe au volant et à ma vie. J'ai peur de l'ennemi pour ma survie et le salut de la patrie. Je le garde en vue, je mets en joue et me camoufle. La

4 Da-sein. Mot-clef de la pensée de Heidegger. Habituellement traduit par être-là. Ce mot veut dire que l'essence la plus originelle de l'humain est d'être le lieu de la manifestation de l'être comme tel. Et dans ce sens se trouve chez l'humain le là de l'être. D'où Da-sein : littéralement là-être; là, il y a être ou manifestation d'être. 
peur est toute ordonnée aux étants qui la provoquent ainsi qu'aux personnes et aux choses pour qui on a peur.

Il en va tout autrement de l'angoisse. Dans l'angoisse on se sent comme à l'étranger et dans un inconfort total parmi les choses qui étaient pourtant jusque-là familières, entourantes et caressantes. On dirait qu'il se produit un décollement par rapport aux étants. C'est comme s'il n'y avait plus de sens où s'orienter, plus de place où poser le pied, plus de havre où accoster, plus de lieu où s'établir et reposer. Toutes choses, y compris l'humain concret qu'est chacun, semblent sombrer dans une sorte d'indifférence diffuse et générale. L'étant dans l'ensemble, pour ainsi dire, recule dans le flou de l'incertain. Mais il ne disparaît pas pour autant. Il se produit plutôt comme une sorte de relâchement des attaches et des rapports aux choses; un relâchement qui laisse notre être flottant dans un vague envahissant et oppressant. Ce recul ou cette retraite de toutes choses qui ne laisse plus aucun appui est la manifestation du rien. Sorte de milieu sans consistance dans lequel semble flotter notre pur fait d'être ou notre pur Da-sein dégagé de tous rapports solides qui rattachent habituellement à la fermeté des choses du monde.

Le rien se montre ainsi dans l'angoisse. Il se montre en même temps que l'étant dans l'ensemble en train de glisser dans l'indifférence totale et retirant tout point d'ancrage et d'inclusion pour notre être-dans-le-monde. Il faut préciser. Le rien se dévoile ou est dévoilé dans l'angoisse, cela veut dire qu'on ne le saisit pas comme on peut saisir un objet. Aussi ne peut-on pas concevoir le rien comme une sorte de chose à côté de l'étant dans l'ensemble; car à côté de celui-ci il n'y a précisément pas de choses ni d'objets. Le rien se montre plutôt à même (in eins mit) l'étant qui dans son ensemble glisse et se dérobe. Ainsi le rien dans l'angoisse n'est ni le résultat de l'anéantissement (Vernichtung) de l'étant, ni l'effet de la négation (Verneinung) de la part de l'entendement humain.

Il importe maintenant de reprendre tout ceci pour dégager plus expressément en quoi consiste l'essence du rien. Pour cela, nous allons suivre encore de plus près le texte de Heidegger (p. 34). Remarquons qu'il s'agit, au fond, de décrire un avènement. Le langage tente tout simplement de montrer, en les étalant, des attitudes qui constituent, somme toute, un seul déploiement. Dans l'angoisse, dit Heidegger, il y a un recul devant... Mais ce recul n'est pas, comme dans le cas de la peur, une fuite plus ou moins éperdue devant un étant déterminé qui s'avère menaçant. Non, ce recul est plutôt un calme ou une retenue, ou encore un arrêt provoqué par la fascination. Ce recul ou ce repli vient du rien ou tire son origine du rien. Car le rien n'attire pas à lui de manière à ce qu'on se lance vers lui pour s'y perdre, mais il est essentiellement répulsif (abweisend) ou renvoyant. Comment comprendre ce renvoi? Comme un 
congédiement? Plutôt comme un laisser prendre congé et, en ce sens, comme une invitation aux préoccupations de tous les jours de suspendre leur cours, de rebrousser chemin en quelque sorte, et de se maintenir en arrêt faute de signification venant de l'ensemble des choses constitutives du monde. Ce renvoi se comprend comme une invitation à retourner sur ses pas ou à se désengager. Mais ce premier renvoi venant de la part du rien, on le voit bien, est comme tel un autre renvoi (Verweisen) qui, lui, concerne l'étant dans l'ensemble en train de sombrer, oriente vers lui tout en le laissant glisser pour ainsi dire vers l'absence. En somme, du rien origine un double renvoi ou un renvoi dans deux directions. Le rien obsédant et oppressant se déploie essentiellement comme un renvoi à l'étant dans l'ensemble en train de se dérober et, en même temps, comme un renvoi qui enjoint de se retenir en arrêt. On voit ainsi que le rien advient de lui-même dans le Da-sein ou en notre être, et non d'autre chose que serait, par exemple, l'intervention de la négation par l'entendement humain.

Si discret soit-il, l'advenir du rien n'est pas un banal événement. Par son double tenvoi signifiant la retenue des implications auprès des choses et montrant l'étant dans l'ensemble en train de glisser dans l'indifférence, il laisse apparaître cet étant dans toute son étrangeté jusque-là assez peu remarquée. Il le laisse apparaître comme le tout autre. Comme le tout autre en face du rien. C'est la manifestation de l'étant en tant que tel ou en tant qu'il est. De là on peut dire qu'il y a de l'étant, et non pas rien, que l'étant est. C'est dans l'advenir originel du rien qu'en tout premier l'être-là ou le Da-sein est amené devant l'étant en tant que tel.

Alors on peut penser l'essence du rien comme le déploiement d'une distance qui permet à l'étant d'apparaître comme tel ou dans son être. Le rien est associé à l'être. Mais l'être de l'étant, ce n'est pas une simple qualité que l'on retrouve tout bonnement chez l'étant parmi d'autres déterminations et qui serait, au même titre qu'elles, observable, analysable, calculable et explicable par les soins de l'une ou l'autre des sciences qui ont cours. L'être de l'étant n'a rien d'étant. Il s'adresse à la pensée dans un autre registre où celle-ci est ouverte à l'autre de l'étant, à ce qui se différencie du tout au tout de l'étant, au non-étant (Nicht-Seiende), au rien de l'étant, en somme, au rien. Là, comme on le voit, se retrouvent ensemble le rien et l'être. Et Heidegger multiplie les formules de ce voisinage essentiel du rien et de l'être. Là, dans ce registre de la pensée, il apparaît que le rien se déploie en tant que l'être.

Nous demandions plus haut si et comment le rien pouvait être considéré comme un joyau pour mériter d'être serré en écrin. On peut voir maintenant que le rien a quelque chose du joyau. Comme lui, il fait apparaître. Le rien fait apparaître l'être. Car le rien s'impose comme le voile 
de l'être. Mais un voile couvre et cache, dira-t-on. La métaphore du voile pour suggérer que le rien fait apparaître l'être serait-elle mal choisie? Mais, faut-il observer, il y a des degrés dans la manifestation. Quelque chose peut apparaître tout en demeurant sous voile, sans être dévoilé expressément. C'est effectivement le propre de ce qui couvre de cacher et de manifester tout à la fois. Un peu comme le secret, d'ailleurs. Que fait le secret? En lui se cache quelque chose; mais cela est quand même manifeste précisément en tant que quelque chose de secret ou gardé en secret. Sans cette sorte de dévoilement, il n'y a tout simplement pas de secret. Tout comme un écrin aussi. L'écrin cache, certes, mais il manifeste déjà en annonçant quelque chose de précieux, de rare. On pourrait dire que l'essence de l'écrin convient à l'essence du rien. Les deux abritent, voilent et manifestent en même temps, gardent en secret. Le rien se déploie comme le secret de l'être même (als das Geheimnis des Seins selbst), dit le texte de départ. En cela il est joyau qui appelle une grandeur et vaut d'être entouré de soins et d'être sauvegardé en écrin.

Mais qu'est-ce qui peut servir d'écrin à ce quelque chose de précieux ou à ce joyau qu'est le rien? C'est la mort, selon la parole de Heidegger : La mort est l'écrin du rien. Il est maintenant temps de lire au complet le paragraphe auquel appartient la phrase qui a servi de départ à notre démarche.

Die Sterblichen sind die Menschen. Sie heißen die Sterblichen, weil sie sterben können. Sterben heißt : den Tod als Tod vermögen. Nur der Mensch stirbt. Das Tier verendet. Es hat den Tod als Tod weder vor sich noch hinter sich. Der Tod ist der Schrein des Nichts, dessen nämlich, was in aller Hinsicht niemals etwas blo $\beta$ Seiendes ist, was aber gleichwohl west, sogar als das Geheimnis des Seins selbst. Der Tod birgt als der Schrein des Nichts das Wesende des Seins in sich. Der Tod ist als der Schrein des Nichts das Gebirg des Seins. Die Sterblichen nennen wir jetzt die Sterblichen - nicht, weil ihr irdisches Leben endet, sondern weil sie den Tod als Tod vermögen. Die Sterblichen sind, die sie sind, als die Sterblichen, wesend im Gebirg des Seins. Sie sind das wesende Verhältnis zum Sein als Sein.

Les mortels sont les humains. Ils s'appellent les mortels parce qu'ils peuvent mourir. Mourir signifie: pouvoir assumer la mort comme mort. Seul l'humain meurt. La bête périt. Elle n'a la mort comme mort ni devant soi ni derrière soi. La mort est l'écrin du rien, à savoir de ce qui à tout point de vue n'est jamais quelque chose de simplement étant, mais qui cependant se déploie, voire même comme le secret de l'être lui-même. La mort, en tant qu'écrin du rien, est l'abri de l'être. Les mortels, nous les nommons maintenant les mortels - non parce que leur vie terrestre prend fin, mais parce qu'ils peuvent assumer la mort comme mort. Les mortels, en tant qu'ils sont ceux qu'ils sont, 
comme mortels, déploient leur essence dans l'abri de l'être. Ils sont le rapport en déploiement à l'être comme être.

Mourir, pour les mortels que nous sommes, c'est pouvoir mourir. Ce n'est pas simplement finir comme finissent les vivants autres que l'humain. Heidegger ne pense pas la mort comme fin de notre vie terrestre. Sans doute parce que, rendu à cette limite précisément, il est déjà trop tard pour dire l'expérience de cette fin et la partager. Mourir, c'est plutôt anticiper la mort, l'assumer, pas seulement et pas d'abord au dernier instant incommunicable de la vie, mais tout au long de son parcours. C'est, à n'importe quel moment, entrevoir, envisager sa propre et ultime possibilité d'exister. La mort est en effet un possible ${ }^{5}$, c'est-à-dire un être que l'on peut, ou encore un pouvoir-être qu'on doit prendre sur soi. La mort est une possibilité absolument propre à chaque Dasein. Il nous est tout à fait impossible de transférer ou de déléguer à d'autres cette possibilité. Elle est aussi incontournable ou certaine. Mais cette certitude reste indéterminée quant au moment. Ce qui fait de la mort une possibilité menaçante sans aucune cesse.

Comment, peut-on maintenant demander, la mort entretient-elle un rapport avec le rien? La mort, comme être-possible, ouvre en quelque sorte une perspective. Celle où le monde dans sa totalité semble couler dans l'absence, où plus rien d'étant ne paraît rester. Mais en même temps que ce rien, surgissent en volte-face ou en contrepartie le fait de l'existence propre et le fait aussi de l'être comme être de tout étant et inséparable de lui. Ainsi la mort laisse apparaître le rien et l'être en virevolte. La mort montre le rien et aussi l'être auquel il est associé.

Maintenant, il reste à expliciter comment la mort peut être considérée comme un écrin. Pouvoir mourir, avons-nous vu, est une possibilité au sens d'une capacité. Une capacité qui peut et qui contient. Qui contient beaucoup. Elle renferme par avance, en effet, la totalité de notre existence ou de notre être-dans-le-monde. Et qu'est-ce que cela comprend? La totalité de nos rapports avec les choses du monde et nos semblables dont nous avons besoin pour exister de fait; la totalité des étants ou l'étant dans l'ensemble au sein duquel nous nous trouvons toujours selon un sentiment de situation donné qui appartient en propre à notre manière d'être. La mort comme pouvoir mourir est cette capacité d'envisager à tout moment de notre existence le possible extrême de cette existence, ou cette existence dans son plein de possible. Et ce plein de possible s'avère l'ultime possible. Ultime comme dernier et comme achèvement. Dans cet ultime possible s'achèvent et prennent fin tous nos rapports avec les étants 
donnés dans l'expérience, y compris nous-mêmes. Alors, en même temps, apparaissent le rien et ce à quoi il renvoie par essence : l'étant dans son ensemble et en tant que tel. Le rien comme joyau éclairant l'étant en tant qu'il est, rehaussant l'être ou l'amenant hors de son retrait. Renfermant tout ceci, la mort peut être considérée comme écrin où se serre ce qui sort du commun, le hors-commun. La mort comme l'écrin du rien, lui-même joyau de l'être.

Mais nous voilà soudain, semble-t-il, devant une difficulté importante. Dans Was ist Metaphysik? Heidegger dit ceci du rien et de l'angoisse :

...die ständige und freilich verdunkelte Offenbarkeit des Nichts, das ursprünglich nur die Angst enthüllt. Darin liegt aber : diese ursprüngliche Angst wird im Dasein zumeist niedergehalten. Die Angst ist da. Sie schläft nur. (p. 37)

... l'ouverture du rien, constante mais certes assombrie, que seule l'angoisse dévoile originellement. Mais ceci implique : cette angoisse originelle est la plupart du temps contenue (retenue, ou tenue dans l'ombre). L'angoisse est là. Elle dort seulement.

Alors l'angoisse est seule à pouvoir faire apparaître le rien, et, en même temps que lui, l'être. Heidegger, cependant, ne dit-il pas lui-même que la mort est écrin du rien et qu'à ce titre elle le manifeste? Et nous avons aussi déjà tenté d'expliciter comment la mort comme ultime capacité d'exister est ouverture du rien et manifestation de l'être. Y a-t-il là une contradiction? Ceci nous oblige à regarder de plus près encore ce qu'implique mourir ou pouvoir la mort.

Mourir, comme pouvoir se projeter à l'avance dans la possibilité d'être la plus propre, possibilité que chacun est seul à pouvoir assumer pour soimême, qui reste incontournable et exige de se libérer pour elle si on veut être pleinement, qui est certaine aussi mais dont le moment reste indéterminé, ce mourir entretient un rapport avec l'angoisse. En effet, précisément en tant que dernier possible indéterminé dans son moment, la mort demeure comme une menace (Bedrohung) constante. Et voici ce que cela implique :

Die Befindlichkeit aber, welche die ständige und schlechthinnige, aus dem eigensten vereinzelten Sein des Daseins aufsteigende Bedrohung seiner selbst offen zu halten vermag, ist die Angst. In ihr befindet sich das Dasein vor dem Nichts der möglichen Unmöglichkeit seiner Existenz. Die Angst ängstet sich um das Seinkönnen des so bestimmten Seienden und erschließt so die äußerste Möglichkeit. Weil das Vorlaufen das Dasein schlechthin vereinzelt und es in dieser Vereinzelung seiner selbst der Ganzheit seines Seinkönnens gewi $\beta$ werden läßt, gehört zu diesem Sichverstehen des Daseins aus seinem Grunde 
die Grundbefindlichkeit der Angst. Das Sein zum Tode ist wesenhaft Angst (SuZ, pp. 265-266).

Mais le sentiment de situation, qui peut tenir ouverte la menace constante et sans restriction s'élevant de l'être du Dasein le plus propre et isolé, est l'angoisse. En elle le Dasein se trouve devant le rien de la possible impossibilité de son existence. L'angoisse s'angoisse pour le pouvoir-être de l'étant ainsi déterminé et ouvre ainsi la possibilité ultime. Parce que l'anticipation (le devancement) rend le Dasein tout à fait singulier et dans cette singularisation de lui-même le fait devenir certain de la totalité de son pouvoir-être, la disposition fondamentale de l'angoisse appartient fondamentalement à ce pouvoir-se-comprendre du Dasein. L'être pour la mort est essentiellement angoisse.

Alors la mort fait planer une menace constante sur notre existence dans son entièreté. Certes, les multiples affairements de la vie de tous les jours peuvent nous amener à oublier cette menace ou à l'ignorer tout simplement. Mais cela n'est pas une manière d'exister très authentique. Car c'est une mise entre parenthèses de notre tâche première qui est d'assumer en propre notre être tout à fait inaliénable, dans son entièreté, jusque dans sa mortalité, dans sa finitude mortelle. Il y a cependant un sentiment de situation ou une disposition profonde qui peut maintenir cette menace à découvert. Et c'est l'angoisse. L'être pour la mort se trouve essentiellement imprégné d'angoisse. Et en tant que tel il est placé devant le rien. Le rien qui pointe avec la cessation apparente de pouvoir tenir tout rapport avec l'étant dans l'ensemble dans l'ultime et dernière possibilité du pouvoir-être du Dasein. Donc l'humain, en tant que Dasein, devant le rien qui surgit dans le devancement ou l'anticipation de la mort comme ultime possibilité, s'angoisse pour son pouvoir-être total ou son incontournable finitude. En ce sens il s'angoisse pour la possible impossibilité de son existence, c'est-à-dire pour cette impossibilité d'exister qu'il a à pouvoir ou qu'il a à assumer.

Ainsi la contradiction que nous avons pu soupçonner à l'instant n'était pas réelle. Si la mort fait aussi apparaître le rien, c'est comme liée essentiellement à l'angoisse. L'angoisse, alors, peut demeurer le seul sentiment de situation qui dévoile l'étrange espace du rien.

L'ouverture du rien, disait aussi Heidegger au même endroit de Was ist Metaphysik?, est constante, mais comme gardée dans la pénombre. L'angoisse, seule disposition ou sentiment de situation à pouvoir ouvrir le rien, est toujours là, mais elle dort. Et comme cette angoisse est liée au devancement (Vorlaufen) dans la mort comme ultime possibilité de notre existence, alors la mort assumée en propre peut tirer l'angoisse de son sommeil. Pas seulement à la fin de la vie, mais tout au long de l'existence. 
Il convient alors de garder en vue l'écrin du rien pour que l'originelle angoisse l'ouvre et laisse apparaître ce rien qui nous gratifie de l'être. Mourir, c'est pouvoir mourir. Et pouvoir mourir, c'est assumer la mort au fil des jours et, par l'angoisse qui lui est inhérente, se tenir dans l'ouverture du rien. Dans ce qui, sous l'espèce du voile, rehausse l'être comme le tout autre et l'inséparable de tout étant quel qu'il soit.

La Postface à la Leçon inaugurale de 1929 intitulée: Was ist Metaphysik?, datée de 1943, fournit de nouvelles indications sur l'angoisse, le rien et l'être. On y reconnaît continuellement des vestiges des monumentales esquisses de la philosophie de l'être publiées dans le tome 65 de l'Édition complète sous le titre Beiträge zur Philosophie ${ }^{6}$. Ces esquisses ont été écrites de 1936 à 1938 et n'ont été publiées qu'en 1989. Elles sont d'un grand secours pour l'interprétation de la pensée heideggérienne dans son ensemble et en particulier pour le thème ici poursuivi.

Alors voici ce que dit cette Postface, entre autres, à propos de l'angoisse :

Denn nahe bei der wesenhaften Angst als dem Schrecken des Abgrundes wohnt die Scheu. (WM, p. 47)

Car dans le voisinage de l'angoisse essentielle en tant que frayeur de l'abîme demeure la déférence.

L'angoisse est Schrecken, frayeur ou saisissement. Mais à proximité du saisissement se trouve la déférence, Scheu. Or ces deux sentiments sont présentés dans Contributions... comme des éléments de la disposition fondamentale (Grundstimmung) de la philosophie de l'autre commencement. C'est-à-dire de la philosophie qui tente de penser l'avènement de l'être même par contraste avec la philosophie du premier commencement qui, dans la disposition fondamentale de l'étonnement, s'est tenue rivée à l'étant pour essayer de le caractériser en tant que tel, donc qui pensait l'être comme qualité ou détermination de tout étant. Et quelle est la disposition fondamentale de l'autre commencement? Heidegger l'appelle la Verhaltenheit. Nous traduisons retenue. Il importe à notre propos de nous y attarder quelque peu. Car pour la pensée de la mort tout autant que pour la

6 Vittorio KlostermanN, Beiträge zur Philosophie (Vom Ereignis). Frankfurt am Main, Gesamtausgabe, 1989, 521 p. Ce livre, pas encore traduit en français, pourrait avoir pour titre: Contributions à la philosophie (De l'avènement). C'est un ouvrage inachevé. Il comprend 281 esquisses plus ou moins longues présentées selon un plan bien précis qui veut indiquer la démarche à suivre par la philosophie de l'autre commencement, i.e. la philosophie qui penserait l'être lui-même en son déploiement plutôt que de s'en tenir à l'étant pour dire en quoi il consiste. 
pensée de l'être et du rien les dispositions et les tonalités affectives sont aussi importantes que la compréhension elle-même. Elles imprègnent la compréhension.

Comme pour la soumettre à une série d'essais de la pensée, cette nouvelle disposition fondamentale reçoit dans un premier temps diverses appellations: Verhaltenheit, Erschrecken, Schrecken, Scheu, Er-ahnen, Ahnung qui est elle-même Schrecken, et Begeisterung : retenue, effroi, saisissement, déférence, pres-sentir, pressentiment ou intuition, et enthousiasme. Mais le mot Verhaltenheit est choisi comme pouvant le mieux rassembler les différents sens qu'évoquent les autres. La Verhaltenheit est finalement présentée comme le style de la pensée originelle dans l'autre commencement et le milieu du saisissement et de la déférence :

Die Verhaltenheit ist die Mitte für das Erschrecken und die Scheu. Diese kennzeichnen nur ausdrücklicher, was ursprünglich zu ihr gehört. Sie bestimmt den Stil des anfänglichen Denkens im anderen Anfang. (B. $65 \S 5$, p. 15)

La retenue est le milieu pour le saisissement et la déférence. Ceux-ci caractérisent seulement plus expressément ce qui lui appartient originellement. Elle détermine le style de la pensée originelle dans l'autre commencement.

Nous allons tenter, dans la perspective de notre thématique de la mort et du rien, une explicitation de cette Verhaltenheit en ayant recours plus ou moins directement à tout ce que nous avons pu glaner dans les 510 pages de ces Contributions....

Nous rendons Erschrecken ou Schrecken par saisissement. On les traduit habituellement par effroi, frayeur, terreur, épouvante. Au plan de la signification, saisissement peut voisiner assez étroitement avec frayeur. Surtout lorsqu'on se garde de ne pas confondre celle-ci avec la peur. La peur fait prendre la fuite et fait chercher un abri ou une protection. Par exemple, la peur d'un chien enragé ou d'un bombardement. Le saisissement, comme la frayeur, se rapproche plutôt de l'ef-froi, ex-fridare. Celuici fait sortir de la paix et, dans ce sens, maintient exposé à ce qui trouble. Dans le saisissement, on est secoué, mais on ne déguerpit pas. Le saisissement, comme l'effroi encore, glace, laisse figé sur place; il fait qu'on s'arrête et qu'on reste bouche bée. Le saisissement coupe la parole. Exemples : saisissement et effroi devant le vide spatial, la puissance nucléaire, la fatalité d'un destin; saisissement et effroi devant la toutepuissance de Dieu, devant la majesté divine. Le saisissement n'est pas loin de la crainte révérencielle et de la déférence. 
De même encore on peut parler du saisissement, de la frayeur et de l'ef-froi devant la mort comme fin de la vie et comme vide de ce qui est familier. C'est alors un bouleversement qui fait sortir de la paix ou de la tranquillité quotidienne, qui trouble la quiétude courante. Devant la mort, on demeure muet, exposé ou sans moyen, comme décontenancé. Lorsqu'elle a frappé dans notre entourage ou que nous nous trouvons dans des circonstances qui la rendent imminente, nous devenons la proie d'un saisissement comme devant quelque chose qui sort du commun. On devient in-quiet fondamentalement. Ce bouleversement et cette inquiétude, il faut insister, se distinguent de la simple agitation intérieure ou de ce trouble psychologique qui amène à se ronger l'esprit et aussi les ongles. Mais ce saisissement, qui n'a rien de maladif, est un dérangement ou un décontenancement : une secousse à la contenance coutumière au sein des choses et des personnes qui nous entourent familièrement, nous supportent et nous protègent.

Et maintenant, Scheu. Nous traduisons par déférence. Elle n'a rien à voir avec la timidité que signifie souvent ce mot. Elle est plutôt parente avec la fascination. Être fasciné, c'est être captivé et attiré. Comme un mouvement vers..., mais qui est tenu en arrêt et en suspens, et, dans ce sens, retenu. La fascination transporte. Mais le transport de la fascination est d'un autre ordre que le changement de lieu dans l'espace. Par exemple: la fascination devant un hypnotiseur, devant une grande beauté, devant l'aventure, devant l'étrange et le dépaysant, devant la vie comme existence donnée en propre et partagée avec d'autres. Il s'agit toujours d'un transport hors du commun, hors de l'attitude habituelle, familière et quotidienne. À cet égard la fascination s'apparente à l'enthousiasme (Begeisterung) qui se retrouve aussi dans la Scheu ou déférence.

Il est bien compréhensible qu'on puisse éprouver de la fascination et de la déférence à l'égard de la mort comme fin de l'existence partagée et comme fin à assumer. Dans la déférence se multiplient les rapports à cette mort : on est captivé, on s'ouvre ou reste exposé, on est maintenu en arrêt ou suspendu, on éprouve comme une attirance ou un besoin d'y voir qui se retient et qui questionne tout à la fois : s'agit-il de la disparition de toute conscience personnelle? de la disparition du monde? de la cessation de l'ouverture dans laquelle tout apparait? de la transmutation de cette ouverture? de la transfiguration de la présence? L'hésitation et le balancement semblent ici régner. L'ambivalence et le vertige. Et le silence aussi. 
Quant à la Verhaltenheit ${ }^{7}$, la retenue, elle rassemble saisissement et déférence. Le mot ne veut pas parler de timidité ni de gêne. Mais il suggère un balancement. D'une part, il laisse entendre une attraction et une propension, mais évoque en même temps un arrêt dans l'élan ou une sobriété. Le mot dit à la fois une échappée et une reprise, un mouvement vers et sa contrepartie. Il évoque la virevolte de mouvements en volteface. La retenue, de plus, suggère l'attitude de l'écoute qui met un frein au flot des mots sur toutes choses. La retenue favorise le nécessaire calme du silence. La retenue peut être considérée comme une synthèse, le milieu où le saisissement et la déférence se rencontrent et s'apparentent.

Ainsi présentée, la retenue est la disposition fondamentale qui permet à l'estre de se déployer ${ }^{8}$. C'est-à-dire qui met l'humain en tant que Da-sein dans l'attitude ou la tenue où il peut entendre la voix silencieuse de l'être et y répondre autrement que dans un discours sur l'étant comme l'a fait la philosophie depuis son commencement. Dans cette tenue de la retenue, le rien peut apparaître comme l'autre de l'étant et le voile de l'être, c'est-àdire, vu la manière typiquement révélatrice du voile, ce par quoi il y a dévoilement d'être. Et il devient ainsi manifeste que cette retenue est la disposition qui permet d'expérimenter la mort comme écrin du rien.

Alors la retenue, par le saisissement qu'elle comporte, englobe l'angoisse puisque celle-ci est aussi saisissement. L'angoisse, dans l'opinion courante, a l'inconvénient d'être un sentiment peu prisé. On redoute l'angoisse parce qu'elle évoque quelque chose qui frôle la morbidité, une attitude ou un tempérament exagérément craintif. On fait des gorges chaudes à propos des "angoisses existentielles " de personnes facilement troublées par tout ou rien. Angoisse est un mot galvaudé, et le sentiment

7 Verhaltenheit. Ce mot ne figure pas au dictionnaire. Heidegger le construit à partir du verbe halten : tenir, arrêter, retenir, maintenir, s'arrêter, etc., ainsi que du verbe verhalten : retenir, contenir, se comporter, se conduire, prendre une attitude. Il y a aussi le nom Verhalten : comportement, manière d'agir. Nous avons choisi de traduire Verhaltenheit par retenue. Parce que ce mot français indique une tenue empreinte de circonspection et de sagesse, de discrétion et de réserve. Aussi parce qu'il peut allier les sens de saisissement et de déférence.

8 Ihr (der Grundstimmung), der Scheu im besonderen, entspringt die Notwendigkeit der Verschweigung, und sie ist das alle Haltung inmitten des Seienden und Verhaltung zum Seienden durchstimmende Wesenlassen des Seyns als Ereignis. (B 65, pp. 15-16) D'elle, (de la disposition fondamentale), de la déférence en particulier, surgit la nécessité du silence, et elle est le laisser-se-déployer de l'estre comme avènement, qui détermine de part en part toute tenue au milieu de l'étant et tout comportement à l'égard de l'étant. Nota bene : Se rappeler que la Grundstimmung, la disposition fondamentale, est la Verhaltenheit, la retenue. 
qu'il évoque d'emblée est bien loin de ce que Heidegger appelle l'angoisse originelle. Par contre, la retenue, synthèse de saisissement et de déférence, et disposition où loge aussi l'angoisse originelle, pourrait être cultivée comme la disposition capable de favoriser la pensée de la mort, la pensée de la mort comme compréhension authentique de l'existence humaine. Disposition fondamentale qui amène devant sa propre mort et la mort des autres comme l'écrin du rien ou joyau de l'être. Et cela journellement. Il serait salutaire de cultiver ou d'entretenir cette retenue pour que les nouvelles de mort, devenues notre lot quotidien, échappent à l'uniformité du classement des simples faits divers et deviennent des annonces audibles du déploiement de l'estre, qui, en dépit du bruit des affairements quotidiens, s'élève silencieusement à travers toutes nos tenues au milieu des étants et dans tous nos comportements à l'égard de ces étants ou de ces choses de la vie courante; et cela parce que nous sommes essentiellement le là de l'être ou Da-sein.

Il reste maintenant à élaborer un rapport suggéré indirectement par les textes de Heidegger entre la mort et l'abîme. Il est d'autant plus pertinent de le faire que ce rapport n'est pas absent des propos de la vie quotidienne. Ne dit-on pas parfois, par exemple, que quelqu'un s'est abîmé dans la mort? Et ne serait-on pas porté aussi à se demander à quoi peut bien servir de s'abîmer dans la pensée du rien et de la mort comme on le fait ici même?

Commençons par situer l'abîme par rapport à l'être et au rien. Voici deux passages suggestifs tirés de Was ist Metaphysik? :

Ohne das Sein, dessen abgründiges, aber noch unentfaltetes Wesen uns das Nichts in der wesenhaften Angst zuschickt, bliebe alles Seiende in der Seinlosigkeit. (WM, p. 46)

Sans l'être, dont le déploiement essentiel, abyssal mais non encore développé, nous est envoyé par le rien dans l'angoisse essentielle, tout étant demeurerait dans la carence d'être.

Le rien dans l'angoisse essentielle nous envoie ou nous fait présent de l'essence abyssale non encore développée de l'être. Le déploiement essentiel de l'être est abyssal, a la propriété de l'abîme. Surprenant. Ainsi se rassemblent angoisse, rien, abîme, être. Comment situer le rien par rapport à l'abîme du déploiement essentiel de l'être, ou par rapport à l'abyssalité de l'être? Et voici cet autre passage qui évoque encore l'abîme :

Eine Erfahrung des Seins als des Anderen zu allem Seienden verschenkt die Angst, gesetzt, daß wir nicht aus "Angst " vor der Angst, $d . h$. in der 
bloßen Ängstlichkeit der Furcht, vor der lautlosen Stimme ausweichen, die uns in den Schrecken des Abgrundes stimmt ${ }^{9}$. (WM, p. 46)

D'une expérience de l'être comme l'autre de tout étant fait don l'angoisse, pourvu que, par "angoisse " devant l'angoisse, i.e. dans la simple anxiété de la peur, nous n'évitions pas la voix insonore qui nous dispose dans le saisissement devant l'abîme.

L'angoisse nous fait le don d'une expérience de l'être. Et comment? Si nous ne passons pas outre à la voix silencieuse qui nous dispose dans le saisissement devant l'abîme. Ce saisissement devant l'abîme, comme nous l'avons montré antérieurement, c'est l'angoisse essentielle elle-même. Angoisse, saisissement devant l'abîme, expérience de l'être se retrouvent dans une même séquence.

Rassemblons: 1. Dans l'angoisse, le rien nous accorde le déploiement abyssal essentiel de l'être. 2. L'angoisse, en tant que saisissement devant l'abîme, nous fait don d'une expérience de l'être. Il y a un rapport certain entre le rien et l'abîme. Mais l'abîme ou l'abyssalité caractérise aussi le déploiement essentiel de l'être. L'abîme est nommé dans ces textes, mais la manière de se rapprocher du rien et de s'articuler à l'être n'est pas explicitée. Qu'est-ce que l'abîme dans la pensée de Heidegger? Quels sont au juste les liens qui le rattachent au rien et à l'être? Et convient-il à la mort? Comment?

Les deux passages tout juste cités sont tirés de la Postface à Was ist Metaphysik?, donc quatorze ans après la Leçon inaugurale de 1929. À michemin dans cet intervalle furent écrites les Contributions... Dans cet ouvrage inachevé, au $\S 242$, pp. 379-388, entre autres lieux, Heidegger tente d'expliciter ce que signifie abîme, Abgrund. Ces pages figurent parmi les plus denses qu'ait écrites le philosophe. Elles sont d'un grand intérêt pour notre propos. Nous nous limiterons cependant à quelques propositions particulièrement significatives.

Der Ab-grund ist die ursprüngliche Wesung ${ }^{10}$ des Grundes. (p. 379)

9 Une voix (Stimme) dispose ou accorde (stimmt). On remarquera la parenté du nom Stimme et du verbe stimmt. Comment, en effet, une voix peut-elle disposer autrement qu'en accordant ou donnant voix? Le langage semble prendre origine dans l'expérience de l'être.

10 Wesung. Mot formé par Heidegger à partir de Wesen qui signifie couramment en philosophie nature, essence, mais qui peut aussi vouloir dire : conduite, manières, façons. Pour Heidegger, il y a une dynamique interne dans l'essence. C'est pourquoi nous traduisons Wesen par déploiement essentiel ou déploiement de l'essence. Wesung 
L'a-bîme est la déployance originelle du fondement.

Ainsi commence le paragraphe. Une proposition énigmatique, s'il en est, mais quand même décisive pour comprendre le sens de Abgrund ou abîme. Plus expressément que notre mot abîme, Abgrund renvoie à fondement, Grund. Les traductions courantes du mot Abgrund sont : gouffre, précipice, abîme. Le mot abîme, lui, vient du latin chrétien abyssus. Celui$\mathrm{ci}$, de son côté, tire son origine du grec $\alpha \beta v \sigma \sigma o s$ ou $\alpha \beta v \theta 0$ s : sans fond, sans limite, immense. Et $\beta v \theta 0 s, \beta v \sigma \sigma o s, \beta v \sigma \sigma \alpha$, veulent dire fond, profondeur, abîme. L'abîme se comprend donc en fonction du fond ou du fondement. Notre mot abîme signifie quelque chose comme une profondeur insondable. Il se dit assez bien des infinis, grand ou petit, et du temps aussi qui paraît insondable dans sa durée en arrière et en avant. Et couramment il évoque la privation de fond ou de fondement ou encore un fond difficile ou impossible à atteindre : a-bîme pour a-fond, a-fondement ou sans fond, sans fondement. Mais dans le contexte présent, a-bîme doit signifier autrement. En séparant le préfixe de la racine du mot, Heidegger, selon son habitude, veut attirer l'attention sur le sens qu'il doit prendre ou sur le rôle précis qu'il est appelé à jouer ici. Quel est ce rôle? Il est indiqué par le a lui-même qui cesse alors d'être simplement privatif. Son nouveau rôle est de dire globalement et succinctement le déploiement de ce qu'est le fondement. Ce mot veut signifier la déployance même de l'essence du fondement, i.e. le fonder. Car le fondement fonde. Alors il faut que soit précisée cette déployance du fondement pour comprendre comment signifie au juste le $A b$ et le $a$ mis en évidence par le trait d'union dans $A b$-grund et a-bîme ${ }^{11}$.

Le propos de l'ouvrage Contributions... est de montrer comment il y a estre ${ }^{12}$. Plus précisément, comment il y a dévoilement d'être chez l'humain, comment se constitue chez l'humain la manifestation de l'être.

veut simplement souligner ce mouvement. Et nous proposons de le rendre par déployance.

11 Le préfixe a vient du grec $\alpha$ et signifie souvent une privation. Mais ce même préfixe a ou ab ou abs est aussi dérivé du latin ab et marque un rapport d'éloignement, d'extraction, de séparation et aussi de privation. Notre contexte va demander de comprendre le ab plutôt dans le sens des rapports d'éloignement et d'extraction.

12 Estre pour être. Heidegger écrit Seyn au lieu de Sein pour attirer l'attention du lecteur sur le fait qu'il s'agit de penser l'être en lui-même et non pas comme étantité de l'étant à la manière de la philosophie traditionnelle. Cette forme ancienne du mot suggère le même procédé en français. Et alors estre s'offre tout naturellement. Sa facture même pourrait aider à comprendre le mouvement qu'il y a dans être et que cette dernière orthographe semble taire. 
Plus succinctement encore, comment le là de l'être se trouve chez l'humain, comment l'être est là, comment il y a Da-sein. C'est dans cette optique qu'il sera question du fondement. Mais l'être, il ne faut pas se méprendre, n'est pas une chose ou un étant que l'on rencontre dans l'humain. L'être n'est pas une chose qui se trouve à l'intérieur d'une autre, à la manière, disons, de l'eau dans un vase. Le là de l'être évoque plutôt la clairière du dévoilement de l'être ou l'éclaircie (Lichtung) de l'être qui advient chez l'humain. Mais l'éclaircie est également le mot qui permet de remonter à l'origine ou au fondement de l' $\alpha \lambda \eta \theta \varepsilon l \alpha$ qui, de son côté, est devenue veritas et vérité au cours de l'histoire de la philosophie. Aussi, peut-on lire ce qui suit au $\S 187$ des Contributions... :

Das ursprüngliche Gründen des Grundes ist die Wesung der Wahrheit des Seyns; die Wahrheit ist Grund im ursprünglichen Sinne.

Le fonder originel du fondement est la déployance de la vérité de l'estre; la vérité est fondement au sens originel.

Comment le fondement fonde-t-il? Selon la déployance de la vérité de l'estre. La vérité, pensée dans son origine, appartient au fondement originel et constitue son mode de fonder. Mais comment se déploie la vérité de l'estre? La vérité ne concerne-t-elle pas les propositions de nos discours? $\mathrm{Ne}$ signifie-t-elle pas l'adéquation de ces propositions aux choses et aux faits auxquels elles se rapportent? Comment peut-il y avoir vérité de l'estre? Avec ces questions, nous revenons au $§ 242$.

La première proposition était : "L'a-bîme est la déployance originelle du fondement. » Après ce que nous venons de dire, il n'est pas étonnant que la deuxième se lise :

Der Grund ist das Wesen ${ }^{13}$ der Wahrheit.

Le fondement est le déploiement essentiel de la vérité.

En court : l'a-bîme est la déployance du fondement, mais le fondement est le déploiement essentiel de la vérité. De sorte que a-bîme et vérité disent la même chose. Il est difficile ici de ne pas oser un rapprochement entre la facture du mot Ab-grund et celle de $\alpha \lambda \eta \theta \varepsilon \imath \alpha$, qu'on a traduit

13 Wesen. Ce mot signifie habituellement en philosophie essence par opposition à existence. Mais la plupart du temps, chez Heidegger, surtout dans les expressions Wesen des Seins ou Seyns, Wesen der Wahrheit, Wesen des Menschen, Wesen des Grundes, Wesen det Sprache, ce mot veut dire en quoi consiste originellement et en eux-mêmes être ou estre, vérité, humain, fondement, langage, et en insistant sur le mouvement qu'il y a dans leur déploiement. D'où notre manière de traduire Wesen par déploiement essentiel, ou parfois tout simplement déploiement. 
traditionnellement par vérité. L' $\alpha-\lambda \eta \hat{\theta} \theta \varepsilon \alpha$, c'est le dé-voilement. Alors on pourrait comprendre $A b$-grund de la façon suivante : de même que la vérité, selon l' $\alpha-\lambda \eta \theta \varepsilon \imath \alpha$, est dé-voilement qui comporte, pour ainsi dire, le mouvement d'une sortie du voilement, et, dans ce sens, un certain à partir du voilement, ainsi, selon le même mouvement, l'Ab-grund indiquerait une sortie du Grund, du fondement, un à partir du fondement. Quelle serait cette sortie qui part de...? Sans doute pas autre chose que le déploiement même du fondement.

Mais il faut tenter d'expliciter davantage, toujours en demandant ce qu'est l'a-bîme :

Was ist der Ab-grund? Welches ist seine Weise des Gründens? Der Ab-grund ist das Weg-bleiben des Grundes.

Qu'est l'a-bîme? En quoi consiste sa manière de fonder? L'a-bîme est le demeurer-à-l'écart du fondement.

L'a-bîme vient de ce que le fondement demeure loin ou à l'écart, comme le weg le suggère. Loin de la surface, semble-t-il, où il y a apparaître et manifestation. Car il ne faut pas oublier qu'il s'agit ici de penser l'advenir de l'estre à son propre. Mais l'être, c'est toujours l'être de quelque étant. L'être et l'étant se différencient, certes, mais selon une différence qui, tout en les distinguant, les tient dans une unité originelle. Il n'y a pas d'étant sans être. Mais ce qui attire d'abord l'attention, c'est l'étant. Celui-ci l'a même toute drainée pendant l'histoire de la philosophie. Et cela grâce à l'être, pourrait-on dire, qui est en quelque sorte demeuré en retrait. Il paraît de la nature de l'être de demeurer à l'écart et de favoriser la manifestation de l'étant. Et en cela même il a le caractère de l'a-bîme. Et il est aussi fondement. Car l'a-bîme, tout demeurer-à-l'écart du fondement qu'il soit, ne supprime pas le fondement.

Alors pour avancer dans la compréhension de l'a-bîme il faut de nouveau se tourner vers le fondement et voir plus expressément comment il fonde.

Und was ist der Grund? Er ist das Sichverhüllende-Aufnehmen, weil ein Tragen, und dieses als Durchragen des Zugründenden. Grund: das Sichverbergen im tragenden Durchragen 14 .

14 Durchragen. Tel quel ce mot n'est pas au dictionnaire. Mais ragen veut dire s'élever, se dresser. Qu'ajoute le durch? Il veut sans doute marquer que le fondement, tout en se cachant, porte et ainsi se dresse en quelque manière au travers de ce qui est à fonder, de l'édifice, par exemple, qui, lui, apparaît nettement. D'où s'élever-au-travers. 
Et qu'est le fondement? Il est le soulever-se-voilant, parce qu'un porter, et celui-ci comme un s'élever-au-travers de l'à-fonder. Fondement : le se-cacher dans le s'élever-au-travers portant.

Il faut paraphraser tout de suite notre traduction très littérale : le fondement est ce qui soulève, mais qui se cache tout à la fois; il soulève parce qu'il porte. Et tout ceci en tant qu'il est ce qui gît au fond et s'élève au travers de cela même qu'il porte. Ce qu'on dit ici du fondement explicite comment et pourquoi l'a-bîme peut être le demeurer-à-l'écart du fondement. La référence aux fondations d'un édifice est assez manifeste. Le fondement est ce qui se cache, ce qui supporte, ce qui soulève; ce qui porte en gisant à la base, ou, dans un sens tout à fait littéral, en étant sous-jacent. Comme sous-jacent, il se cache, demeure à l'écart. Mais le fondement est fondement pour quelque chose. Il a un être ordonné à ce qui est à fonder. Il se manifeste donc dans cela et au travers de cela à quoi il est ordonné comme supportant. Et ce serait en cette manière que le se-cacher ou le demeurer-à-l'écart du fondement est signifié ou manifesté. Ainsi l'Ab-grund, l'a-bîme, comme tenue-à-l'écart du fondement, nommerait simplement la manière qu'a le fondement de fonder, de porter, en se cachant certes, mais tout en se montrant en même temps dans ce qu'il supporte.

Mais la manière qu'a le fondement de se cacher peut être précisée :

Ab-grund das Ausbleiben; als Grund im Sichverbergen, ein Sichverbergen in der Weise der Versagung des Grundes. Versagung aber ist nicht nichts, sondern eine ausgezeichnete ursprüngliche Art des Unerfüllt-, des Leerlassens; somit eine ausgezeichnete Art der Eröffnung.

A-bîme le demeurer en dehors; comme fondement dans le se-cacher, un se-cacher selon la manière du refus du fondement. Refus cependant n'est pas rien, mais une manière originelle insigne du laisser non rempli, du laisser vide; ainsi une manière insigne de l'ouverture.

L'a-bîme, c'est le demeurer en dehors ou le ne pas venir en avant du fondement. C'est le fondement en tant qu'il se cache; et le fondement se cache selon sa tenue en retrait qui a elle-même l'allure d'un refus. Pour comprendre la portée de ce refus, il faut se rappeler qu'on tente de dire comment l'estre advient au propre de lui-même tout en rendant compte pourquoi et comment l'être n'a pas été pensé expressément pour lui-même jusqu'à maintenant. Il ne s'agirait pas d'une simple mégarde, mais cela relèverait de la nature même de l'être. L'estre semble comporter un refus. Or le refus n'est pas une nullité ou un simple négatif. Il s'avère plutôt une façon spéciale de laisser non rempli, de ne pas occuper la place ou de laisser vide. De la sorte le refus est une manière originelle d'ouvrir. Une ouverture tout à fait remarquable. 
Et le texte continue. Il reprend succinctement les acquis, en en soulignant des aspects, pour ensuite porter plus loin l'explicitation de l'a-bîme:

Allein, der Ab-grund ist als Wesung des Grundes kein bloßes Sichversagen als einfacher Rückzug und Weggang. Der Ab-grund ist Ab-grund. Im Sichversagen bringt der Grund in einer ausgezeichneten Weise in das Offene, nämlich in das erst Offene jener Leere, die somit eine bestimmte ist. Sofern der Grund auch und gerade im Abgrund noch gründet und doch nicht eigentlich gründet, steht er in der Zögerung.

Seulement, l'a-bîme comme déployance du fondement n'est pas un pur se-refuser comme simple retraite ou départ. L'a-bîme est a-bîme. Dans le se refuser le fondement amène d'une manière insigne dans l'ouvert, à savoir dans l'ouvert premier de ce vide, qui ainsi est un vide déterminé. Dans la mesure où le fondement, aussi et précisément, fonde encore dans l'abîme et cependant ne fonde pas proprement, il se tient dans l'hésitation.

L'a-bîme est un refus. Mais il faut se garder de comprendre ce refus comme un se refuser total, comme une retraite ou un départ pur et simple. En effet, l'a-bîme veut dire la déployance du fondement. Dans l'a-bîme, le fondement est toujours là et, même à l'écart, il continue de fonder. Mais son fonder comporte un refus. En se refusant le fondement amène d'une manière insigne dans l'ouvert, à savoir dans l'ouvert de ce vide déterminé ou particulier que le refus même ouvre ou laisse béant. Qu'entendre par cette ouverture d'un vide déterminé? Déterminé parce que marqué par le refus lui-même. Quelle est cette marque? La marque de ce qui s'annonce de quelque manière et qui se retient cependant. Ainsi le fondement, en tant qu'il fonde en se refusant, se tient dans une sorte d'hésitation. C'est vers cette idée d'hésitation que s'en allait tout cet alinéa. Elle est importante. Heidegger la reprendra abondamment dans ses ouvrages ultérieurs. Ici elle évoque comment, en tant qu'a-bîme, le fondement continue de fonder mais, cependant, pas tout à fait proprement. C'est-à-dire? Il continue de fonder, mais pas expressément ou sans être vu comme tel immédiatement, justement en tant que demeurant à l'écart et dans ce sens se refusant. Et, en général, cette idée d'hésitation renvoie à l'être qui, certes, est toujours associé à l'étant, mais qui, laissant pour ainsi dire le devant de la scène à ce dernier, se tient en retrait, tout en continuant quand même de se manifester discrètement comme l'être plus ou moins oublié de l'étant ou encore dans la reconnaissance plus claire que l'on a, de temps en temps, du fait qu'il n'y a pas d'étant sans être.

Ainsi en résumé : l'a-bîme est la tenue à l'écart du fondement; il dit que le fondement fonde en se cachant selon la manière du refus. Or serefuser laisse un vide, le vide de ce qui s'est annoncé et toutefois retenu, 
donc, en ce sens même, une ouverture déterminée, marquée par l'hésitation.

Maintenant un pas de plus qui va permettre de mieux entrevoir le rapport entre a-bîme et vérité :

$A b$-grund ist die zögernde Versagung des Grundes. In der Versagung öffnet sich die ursprüngliche Leere, geschieht die ursprüngliche Lichtung, aber die Lichtung zugleich, damit sich in ihr die Zögerung zeige.

L'a-bîme est le refus hésitant du fondement. Dans le refus s'ouvre le vide originel, advient l'éclaircie originelle, mais l'éclaircie en même temps afin qu'en elle se montre l'hésitation.

On a explicité jusqu'ici que l'a-bîme, déployance essentielle du fondement, comporte un refus hésitant du fondement, que ce se-refuser n'est pas un simple départ, qu'il amène plutôt dans l'ouvert de ce vide que constitue justement le se-refuser. Il s'agit d'un vide originel parce que se trouvant au plan du fondement. Ce vide est appelé clairière ou, mieux encore, l'éclaircie originelle. Elle advient en même temps que le se refuser ou à la fois, de manière à ce que le mouvement typique de l'hésitation, son vacillement, se montre en elle. Pour bien comprendre il faut garder en mémoire qu'on tente ici d'expliciter comment s'ouvre originellement, donc tout d'un coup et sans recours à autre chose d'antérieur, la toute première éclaircie, Lichtung. On traduit habituellement Lichtung par clairière. Mais éclaircie semble particulièrement apte à dire le dégagement qui advient dans le se-refuser hésitant du fondement. Le mot éclaircie dit que la clairière comporte à demeure un éclaircir, une sorte de dégagement constant. Éclaircie comme résultante toujours à conquérir de l'éclaircir incessant que comporte le balancement du se-refuser hésitant. C'est que l'origine ne doit pas être conçue comme un point de départ que l'on quitte ou laissse en arrière. L'origine advient et se maintient comme origine dans le maintien de sa déployance ou dans son incessante déployance.

Et maintenant on reprend le tout :

Der Abgrund ist die erstwesentliche lichtende Verbergung, die Wesung der Wahrheit.

L'a-bîme est le premier essentiel cacher éclaircissant, la déployance de la vérité.

Cet alinéa résume magistralement la démarche accomplie. Il s'agissait de montrer que l'a-bîme, déployance originelle du fondement, est le déploiement de l'essence de la vérité. Et c'est ce qui s'est produit en explicitant le fonder du fondement selon le se refuser hésitant qui amène, en tant que tel, dans l'ouvert du vide, ou dans l'éclaircie. Celle-ci est marquée 
essentiellement par le vacillement de l'hésitation. Et en tant que telle elle est l'éclaircie du se refuser qui à la fois cache et amène dans l'ouvert. Mais l'éclaircie n'est pas une éclaircie produite une fois pour toutes. On a donc plutôt affaire à un se-cacher dégageant, un cacher é-claircissant. Le cacher et l'éclaircir n'ont pas de cesse; ils sont comme dans une joute incessante. Voilà la déployance originelle de l'essence de la vérité. Et voilà la déployance originelle du fondement en tant qu'a-bîme.

Une autre étape, enfin, pour montrer le rapport entre l'a-bîme et l'être. Rapport que nous n'avons d'ailleurs cessé d'évoquer par avance afin de mieux voir le sens de la démarche.

Da aber die Wahrheit die lichtende Verbergung des Seyns ist, ist sie als Abgrund zuvor Grund, der nur gründet als das tragende Durchragenlassen des Ereignisses. Denn die zögernde Versagung ist der Wink, in dem das Da-sein, eben das Beständnis der lichtenden Verbergung, erwunken wird, und das ist die Schwingung der Kehre zwischen Zuruf und Zugehörigkeit, die $\mathrm{Er}$ eignung, das Seyn selbst.

Mais comme la vérité est le cacher éclaircissant de l'estre, elle est comme a-bîme tout d'abord fondement, qui fonde seulement en tant que le laisser-s'élever-au-travers portant de l'avènement. Car le refus hésitant est le signe dans lequel l'être-là, précisément la contenance du cacher éclaircissant, est signalé, et ceci est le balancement de la volte-face entre l'appel-à et l'écoute-appartenance ${ }^{15}$, l'a-venue, l'estre même.

Donc un pas plus loin dans la pensée de l'abîme : la vérité reconduite à la source de sa provenance aléthéienne est la vérité de l'estre. Elle est le cacher éclaircissant ou dégageant de l'estre. Alors on doit dire ceci : en tant qu'a-bîme la vérité est d'abord fondement; et ce fondement ne fonde seulement qu'en tant qu'il porte et laisse s'élever l'avènement (Ereignis), i.e. l'avènement de l'estre à son propre ou au propre de luimême. Un avènement marqué du vacillement de l'hésitation.

Alors il faut comprendre le se refuser hésitant du fondement, amenant dans l'ouvert et s'avérant ainsi une ouverture originelle, et qui est en cette manière le cacher éclaircissant, il faut le comprendre comme un

15 Zugehörigkeit : écoute-appartenance. Zuhören veut dire écouter. Zugehörigkeit signifie d'habitude tout simplement appartenance. Mais comme Heidegger place ce mot en vis-à-vis avec Zuruf qui signifie appel, il est tout à fait convenable de penser qu'il charge Zugehörigkeit des deux sens : appartenance et écoute. À noter, en passant, que Heidegger voit dans cet appel et cette écoute-appartenance dans l'estre l'origine même du langage. 
signe ou un faire signe de l'estre. C'est dans ce signe qu'il y a Da-sein ou là-être. C'est dans ce signe que s'ouvre le là de l'être. Et ce là de l'être, assumé par l'humain, soutient cette éclaircie en prenant pour ainsi dire la contenance (Beständnis) de ce cacher éclaircissant et en en assurant la tenance. À cet égard l'humain est le tenant ou le tenancier de l'estre. L'être-là ou le là de l'être surgit dans l'humain comme le signalement de l'estre. C'est selon cette tournure qu'advient l'avènement (Ereignis), que se produit l'ad-venue à son propre (Er-eignung), son a-venue ou a-venance. Mais tout cela, c'est l'estre même.

L'a-venue (Er-eignung) de l'a-vènement (Er-eignis) est appel-à (Zuruf) et écoute-appartenance (Zugehörigkeit). Appel-à qui va vers l'être-là et écoute-appartenance qui va vers l'estre. Mais il faut prendre garde dans cette dernière formulation de ne pas chosifier être-là et être; il faut éviter de les considérer comme étant deux choses, chacune de son côté, antérieurement au mouvement en volte-face de l'appel-à et de l'écoute-appartenance. Cependant on doit y voir l'origine du langage chez l'humain. L'estre est le balancement de la volte-face ou de la virevolte (Kehre, tournant) entre l'appel-à ou le faire signe et l'appartenance-écoute ou la tenance. La même a-venue chez l'humain d'une interpellation à être le là et d'une réponse qui y correspond selon l'appartenance d'origine. L'estre est cet avènement.

C'était notre incursion dans Contributions... Nous avons vu se préciser le rapport entre l'a-bîme et l'être. Nous pouvons maintenant mieux comprendre comment l'être est abyssal. Mais quelle sorte d'éclairage avonsnous obtenu sur le rapport entre l'abîme et le rien? Car c'était aussi notre question. Voilà : nous pouvons penser maintenant qu'il faille reconnaître le rien dans le vide originel, l'éclaircie originelle, surgissant du refus hésitant du fondement ou de l'a-bîme. Et le rien de cet a-bîme appartient à l'avènement même de l'estre. Le rien est partie prenante de l'être. Il s'ouvre comme a-bîme de son déploiement. Il y a du rien dans l'être. Le rien de l'abîme de l'être.

Et cette interprétation paraît conforme à ce que dit Heidegger luimême au tout début de Contributions...

...oder kann die Verweigerung (das Nichthafte des Seyns) im Äußersten zur fernsten Er-eignung werden, gesetzt, daß der Mensch dieses Ereignis begreift und der Schrecken der Scheu ihn in die Grundstimmung der Verhaltenheit zurück- und damit schon in das Da-sein hinaustellt? (B 65, p. 8)

... ou le refus (le caractère du rien de l'estre) peut-il à l'extrême devenir la plus lointaine a-venance, à supposer que l'humain saisisse cet avènement et que le saisissement de la déférence le ramène dans 
la disposition fondamentale de la retenue et ainsi déjà l'expose dans le là de l'être.

Le refus, que l'on sait maintenant appartenir à l'a-bîme et qui laisse s'ouvrir le vide originel, est ici expressément présenté comme ayant le caractère du rien logeant lui-même dans l'estre. Il est donc légitime de voir le rien dans le vide du refus hésitant du fondement, i.e. dans l'abîme.

Conforme aussi à cet autre passage de la Postface de 1943 déjà évoqué :

... im Nichts die Weiträumigkeit ${ }^{16}$ dessen zu erfahren, was jedem Seienden die Gewähr gibt, zu sein. Das ist das Sein selbst. Ohne das Sein, dessen abgründiges, aber noch unentfaltetes Wesen uns das Nichts in der wesenhaften Angst zuschickt, bliebe alles Seiende in der Seinlosigkeit. Allein auch diese ist als die Seinverlassenheit wiederum nicht ein nichtiges Nichts, wenn anders zur Wahrheit des Seins gehört, daß das Sein nie west ohne das Seiende, daß niemals ein Seiendes ist ohne das Sein. (WM, p. 46)

...expérimenter dans le rien la large spatialité qui donne à tout étant la garantie d'être. Cela c'est l'être même. Sans l'être, dont le déploiement abyssal mais encore non-développé nous est gratifié par le rien dans l'angoisse essentielle, tout étant demeurerait dans la carence d'être. Seulement, même celle-ci comme abandon par l'être n'est pas un rien négatif, si en effet appartient à la vérité de l'être que l'être ne se déploie jamais sans l'étant, qu'un étant n'est jamais sans l'être.

Dans le rien on peut expérimenter le vaste espace qui permet à tout étant d'être. Ce vaste espace est le vide originel ou l'éclaircie de l'estre. Mais celle-ci surgit comme déployance même du fondement ou comme abîme. Alors dans le rien on peut expérimenter l'a-bîme et les deux sont étroitement associés à l'estre. Le rien est comme l'accès à l'a-bîme et à l'être.

Et qu'en est-il, enfin, du rapport de la mort à l'abîme? En sa qualité d'écrin du rien elle se trouve à introduire à l'abîme. À l'abîme de l'estre. $\grave{A}$ cette ouverture où l'humain peut se comprendre comme le dépositaire du déploiement de l'être pour la manifestation de toutes choses comme étants.

16 La large spatialité ou le vaste espace ici mentionné évoque l'espace-temps (ZeitRaum) en quoi culmine l'analyse de l'a-bîme au $\S 242$ de Contributions... Nous manquons ici précisément d'espace et de temps pour suivre cette démarche jusqu'au bout. Sans compter que ce bout n'en est sans doute pas un. 
Nous devons maintenant conclure. La mort est l'écrin du rien s'avère une parole étrangement chargée de sens. L'humain est essentiellement marqué par la mortalité. La mort est son pouvoir être suprême. Alors cette parole sur la mort peut nous disposer à la retenue en qui se rejoignent le saisissement propre à l'angoisse originelle devant le rien de l'a-bîme de l'être, ainsi que la déférence pour l'avènement de celui-ci à lui-même par le recours au Da-sein humain. Et qu'est-ce que tout ceci peut signifier pour la pensée à la fin de notre parcours? Comment ceci peut-il interpeller la pensée qui a voulu cheminer jusqu'ici? C'est peut-être cela que Heidegger avait en vue quand il écrivait en $1954^{17}$ :

Die Forderung erscheint in einem seltsamen Licht, wenn wir uns darauf besinnen, daß das Wesen der Sterblichen in die Achtsamkeit auf das Gehei $\beta$ gerufen ist, das sie in den Tod kommen heißt. Er ist als äußerste Möglichkeit des sterblichen Daseins nicht Ende des Möglichen, sondern das höchste Gebirg (das versammelnde Bergen) des Geheimnisses der rufenden Entbergung.

L'exigence apparaît dans une lumière étrange quand nous méditons ceci, à savoir que l'essence des mortels est convoquée à rendre attention à l'appel qui les invite dans la mort. Elle n'est pas, comme extrême possibilité du Dasein mortel, la fin du possible, mais le suprême abri (la mise à l'abri rassemblante) du secret du dévoilement appelant.

Dans cette dernière expression dévoilement appelant, nous retrouvons le cacher dégageant ou éclaircissant de l'estre (die lichtende Vergergung) qui comporte un appel (Zuruf) à la tenue dans le là de l'être et une écouteappartenance (Zugehörigkeit) dont il fut question auparavant. L'extrême possibilité du là est d'abriter, de rassembler au mieux ou au maximum le secret (des Geheimnisses) de l'estre qui est appel du désabritement (der rufenden Entbergung). L'humain est appellé à ce sommet du possible. Cette extrême possibilité est la mort. L'humain est appelé à la mort. Mais on voit bien que la mort, précisément comme abritement du secret de l'être, n'est pas la fin du possible. Expressément pensée, elle s'avère plutôt la tenue où l'estre peut révéler au mieux son secret : son accointance obligée avec l'essence originelle de l'humain en quoi celui-ci se révèle à lui-même comme le veilleur de la vérité de l'estre. Nous reprenons ainsi la parole initiale : la mort est l'écrin du rien comme joyau de l'abyssalité de l'être ou de sa vérité.

Voilà ce qui est enjoint à la pensée. Disposée selon la retenue, son destin est d'accepter d'être transportée dans l'achèvement du possible qu'est la mort, et là, de se laisser saisir par le large du rien de toutes 
choses et en même temps de se laisser emporter dans la déférence pour l'être, ce tout autre qui se distingue des étants tout en leur demeurant indissolublement lié et que le rien voile et annonce à la fois. Tout au long de l'existence la pensée est invitée à s'adonner au secret de l'abyssalité de l'estre contenue dans la mort. L'achèvement ou l'accomplissement du possible qu'est la mort comme possible ultime n'est pas la fin du possible. Mais c'est le projet d'une tâche toujours à reprendre en pensée.

On peut comprendre cela tant que la vie dure. Mais la mort, au moins selon les apparences, évoque quand même une fin, celle de la vie. Et la pensée au bord de cetre limite se trouble et s'inquiète. Sur cette frontière et au-delà d'elle qu'advient-il, se demande-t-elle, de la manifestation du rien et de l'être? Qu'advient-il d'elle-même?

Le passage que nous venons de citer est précédé dans le texte par la proposition suivante qui peut être éclairante à cet égard :

Wer jedoch vom Denken nur eine Versicherung erwartet und den Tag errechnet, an dem es ungebraucht übergangen werden kann, der fordert dem Denken die Selbstvernichtung $a b$.

Qui cependant attend de la pensée seulement une assurance et escompte le jour où, non requise, on pourra se passer d'elle, réclame de la pensée qu'elle s'anéantisse elle-même.

Alors reposons notre question: qu'arrive-t-il à la fin de l'ultime possible, c'est-à-dire à la mort comme fin de la vie? Comment le savoir? Comment le savoir sans demander si ce que nous appelons la fin de la vie peut coïncider avec la fin de l'ultime possible? Comment savoir en quoi consiste la fin d'un possible, surtout quand il est l'ultime, le suprême? A-t-il tout simplement une fin? Y a-t-il une science qui peut nous en informer? Il y a peut-être la foi qui offre à cet égard quelque certitude. Mais alors le divin est sans doute entré en ligne de compte. Et comment le divin peut-il se manifester pour la pensée du rien, de l'abîme et de l'être? Entretenir ces questions, c'est justement ne pas donner congé à la pensée de l'estre et de son abyssalité. Mais c'est en même temps renoncer à une assurance qui, en neutralisant la retenue, empêcherait les mortels que nous sommes d'être à la fois les sentinelles du rien ${ }^{18}$ et les bergers de l'être ${ }^{19}$. Comme là de l'être (Da-sein), l'humain hérite essentiellement de l'hésitation de l'estre et demeure, en sa pensée, soumis au vacillement des composantes de la retenue originelle. Saisissement et déférence. Une inquiétude salutaire.

Voir Was ist Metaphysik?, p. 38; Zur Seinsfrage, p. 38 : Platz-halter des Nichts.

19 Voir Brief über den Humanismus, GA, 9, p. 328 et 338 : Der Mensch ist der Hirt des Seins. 